\title{
SELECTION OF DROUGHT TOLERANT WHEAT GENOTYPES BY OSMOTIC STRESS IMPOSED AT GERMINATION AND EARLY SEEDLING STAGE
}

\author{
R.R. Saha ${ }^{1 *}$, A. Hannan ${ }^{2}$, A. Nessa ${ }^{2}$, M.A. Malek ${ }^{3}$ and M.R. Islam ${ }^{4}$ \\ ${ }^{1}$ Plant Physiology Division, Bangladesh Agricultural Research Institute, Gazipur-1701, Bangladesh \\ ${ }^{2}$ Seed Technology Division, Bangladesh Agricultural Research Institute, Gazipur-1701, Bangladesh \\ ${ }^{3}$ Plant Genetic Resources Center, Bangladesh Agricultural Research Institute, Gazipur-1701, Bangladesh \\ ${ }^{4}$ Regional Agricultural Research Station, BARI, Ishurdi, Pabna, Bangladesh
}

\begin{abstract}
An experiment on hundred wheat genotypes under different levels of osmotic stress was carried out during 2014 to select the genotype(s) tolerant to drought at germination and early seedling stage. Different levels of osmotic stress were imposed by using polyethylene glycol (PEG). Three osmotic stress levels viz. control (distilled water), 15\% PEG solution and 25\% PEG solution were used. Among the 100 genotypes the rate of germination percentage, final germination (\%), root and shoot dry weight, amount of respiration and vigour index under PEG treatment was found significantly lower than that of control condition. Compared to control condition relative decrease in rate of germination, final germination, amount of respiration and vigour index among the wheat genotypes were found more at $25 \%$ PEG than that of $15 \%$ PEG treatment. However, the seed metabolic efficiency was significantly higher in wheat genotypes under both 15\% PEG and 25\% PEG treatment compared to the control condition. A significant positive correlation exists between the important growth parameters like rate of germination (\%), final germination (\%), shoot dry weight, root dry weight and vigour index. On the basis of these physiological traits against osmotic stress, nine genotypes of wheat such as BD-480, BD-498, BD501, BD-513, BD-514, BD-519, BD-592, BD-618 and BD- 633 were selected as drought tolerant.
\end{abstract}

Keywords: Drought tolerant, osmotic stress, wheat genotypes

\section{INTRODUCTION}

Wheat is one of the most important cereal crops of the world. In most areas of the world, wheat is a principal food. In Bangladesh, it is the second most important grain crop after rice and grown in winter season which prevailing drought condition due to

\footnotetext{
"Corresponding author e-mail: saharinarani@yahoo.com
} 
lack of rains. Drought is a severe limitation of plant growth, development and productivity, particularly in arid and semi-arid regions (Galle et al., 2007). Seed germination and early seedling growth are potentially the most critical stages for water stress (Ahmad et al., 2009). Besides the reduction in total germination, comparatively low soil moisture availability results in delayed emergence, a criterion of particular importance in the vigor and subsequent yielding ability of many crops (Azam and Allen, 1976). The rate and degree of seedling establishment are extremely important factors in determining both yield and time of maturity (Brigg and Aylenfisu, 1979).

Abiotic stresses like drought can directly or indirectly affect the physiological status of an organism by altering its metabolism, growth, and development. Among these, drought is a worldwide problem, constraining global crop production seriously and recent global climate change has made this situation more serious (Pan et al., 2002). Plants respond to drought with physiological and biochemical changes. The impacts of drought condition depend on their severity and the stage of plant growth during which they occur. Seedling emergence is one of the most sensitive growth stages that are susceptible to water deficit. Therefore, seed germination, seedling vigour and coleoptiles's length are prerequisites for successful stand establishment of wheat under drought conditions. Simulation of drought stress by polyethylene glycol (PEG) induces drought stress on the plants (Jiang et al., 1995). PEG-6000 has long been utilized as a reliable marker under laboratory conditions for testing the drought tolerant genotypes which could be a cost effective and still potential approach. With this view the present study was conducted to select wheat genotype(s) for their drought tolerance at the germination and early seedling stage by using PEG induced water stress.

\section{MATERIALS AND METHODS}

The experiment was conducted in both the laboratories of Plant Physiology Division and Seed Technology Division, BARI, Gazipur during 2014. The experiment was laid out in a completely randomized design with two replications. Hundred wheat genotypes were tested under osmotic stress imposed by using polyethylene glycol (PEG6000). Solutions were prepared according to Baloch et al. (2012). There were three osmotic stress levels i.e. $\mathrm{T}_{1}$-control (with distilled water), $\mathrm{T}_{2}-15 \%$ PEG solution and $\mathrm{T}_{3} 25 \%$ PEG solution. Thirty seeds were placed in Whatman number 1 filter paper in petridishes. After placing the seeds in petridishes, measured volume of $10 \mathrm{ml}$ PEG solution or distilled water was given in the petridishes. Three days after placement of seeds in the petridishes, $10 \mathrm{ml}$ distilled water was added in each petridishes to minimize the evapo-transpiration losses. Germination was counted at 24-hour interval and continued up to the 7 day. The seeds were considered germinated when plumule and radicle came out and longer than $2 \mathrm{~mm}$. After 10 days of treatment, other parameters such as number of seedling, dry weight of shoot, root and remaining seed was taken on all seedlings of each treatment which having at 
least $3 \mathrm{~cm}$ long in both root and shoot. The rate of germination was calculated using the formula according to Krishnasamy and Seshu (1990). Seed metabolic efficiency (SME) and amount of seed material respired (SMR) were calculated using the formula according to Rao and Sinha (1993). All percentage data were transformed into arcsine value and recorded data were analyzed statistically (ANOVA and correlation). Least Significant Difference (LSD) was used to compare the mean differences among the treatments.

\section{RESULTS \& DISCUSSION}

\section{Different seedling traits}

Varying response of wheat genotypes to PEG treatment is very important for screening drought tolerant genotypes at early seedling stage before conducting extensive and expensive field tests. In the present study, rate of germination (\%), final germination (\%), root and shoot dry weight, amount of respiration of 100 wheat genotypes were significantly decreased due to osmotic stress i.e. PEG treatment compared to that of control treatment (Table 1 \& 2). Roza et al. (2010) noted that significant decrease in shoot and root dry matter of wheat at PEG treatment. On the other hand, dry weight of remaining seed, root-shoot ratio and seed metabolic efficiency were significantly increased at PEG treatment than that of control condition (Table 2). It was observed that the range between maximum and minimum values of rate of germination (\%) and final germination (\%) among the 100 wheat genotypes under PEG treatment was higher than that of control treatment. It might be due to variable responses of wheat genotypes to osmotic stresses. It was also found that the rate of germination and final germination relatively decreased more in $25 \%$ PEG than that of $15 \%$ PEG treatment. This was probably due to depression in traits due to PEG desiccation. The remaining seed dry weight was higher in both $15 \%$ PEG and 25\% PEG treatment than that of control treatment. The result indicated that PEG induced stress condition might be affected the transformation of seed reserve to available form which was essential for producing seedling organ i,e, root and shoot. It is also observed that dry matter was more or less equally distributed in root, shoot and remaining seed but slightly higher dry matter utilized for respiration under control condition. Whereas, in case of PEG treatment, most of the dry matter remained in seeds which might be due to inhibitory effect of osmotic stress on breakdown of seed reserves resulting lower accumulation of root and shoot dry matter as well as utilizing smaller amount of dry matter for respiration. For this reason, decreasing trend was presumably found in seed germination as well as seedling growth.

Root and shoot ratio significantly varied among the wheat genotypes under different level of stress (Table 2). Higher root-shoot ratio was observed in all the genotypes under drought stress condition i.e. PEG treatments compared to control condition. This might be due to the tendency of enhanced root growth for surviving under stress 
condition. The development of root system under water deficit conditions appears to be a very viable criterion to select water stress tolerant genotypes of a crop because the roots take the moisture from lower layers of soil. Dhanda et al. (2004) reported that continued growth of roots in dry soil is particularly important to avoid drought stress. On the other hand, significantly decreasing amount of respiration was found in all the genotypes under PEG treatment than that of control condition which indicated a lower enzymatic activity under stressful conditions. However, the seed metabolic efficiency was significantly higher in wheat genotypes under both $15 \%$ PEG and $25 \%$ PEG treatment compared to the control. Under stress condition, the higher value of seed metabolic efficiency indicated the seeds may have higher efficiency to utilize seed reserves for producing root and shoot rather than respiration and/or unable to breakdown the seed reserve resulting higher remaining seed dry weight.

\section{Vigour index}

Vigour index differed significantly among the wheat genotypes under variable drought condition (Table 3). Due to PEG treatment considerable reduction was found in seed vigour index in all the genotypes compared to control condition. Relatively more reduction of vigour index was observed among the wheat genotypes at $25 \%$ PEG than that of 15\% PEG treatment. Similar to present findings, Bayoumi et al. (2008) and Rauf et al. (2007) also noted significant reductions in all seedling traits by osmotic stress in wheat. However, among the 100 genotypes, nine genotypes such as BD-480, BD-498, BD-501, BD-513, BD-514, BD-519, BD-592, BD-618 and BD633 showed relatively less reduction in vigour index at both $15 \%$ PEG and 25\% PEG treatment and these genotypes were regarded as drought tolerant.

\section{Correlations among seedling traits}

Correlations are important statistical parameters for selection and crop improvement program (Baloch et al. (2012). Rate of germination showed significantly positive correlation with shoot dry weight $(\mathrm{r}=0.64)$, amount of respiration $(\mathrm{r}=0.46)$ and vigour index $(\mathrm{r}=0.6)$ but negative correlation with remaining seed dry weight $(\mathrm{r}=-$ $0.57)$ and root-shoot ratio $(r=-0.39)$ (Table 4). Final germination $(\%)$ and root dry weight expressed significantly positive correlation with vigour index $(r=0.78$ and 0.7 , respectively). Shoot dry weight showed significantly positive correlation with amount of respiration $(r=0.54)$ and vigour index $(r=0.81)$ but significantly negative correlation with remaining seed dry weight, total seedling dry weight and root-shoot ratio. In case of remaining seed dry weight, significant positive correlation was found with total seedling dry weight $(r=0.88)$ but significantly negative correlation with amount of respiration $(\mathrm{r}=-0.64)$ and vigour index $(\mathrm{r}=-0.65)$. Total dry weight of seedling positively correlated with seed metabolic efficiency $(r=0.48)$ and negatively correlated with amount of respiration $(r=-0.56)$. Similarly amount of respiration showed significantly negative correlation with seed metabolic efficiency(r $=-0.8)$. Moreover, rate of germination $(\%)$, final germination $(\%)$, root and shoot dry weight and vigour index exhibited significant positive correlations with each other, 
which suggesting that increase in any one of those traits correspondingly increase the other traits. It means that if one reliable trait is picked in osmotic stress and used as selection criterion that will lead to improve other seedling traits for drought conditions (Baloch et al., 2012). Several other workers (Bayoumi et al., 2008, Dhanda et al., 2004, Rauf et al., 2007 and Baloch et al., 2012) also noted positive correlations among wheat seedling traits under osmotic or water stress condition.

Table 4. Correlation coefficient (r) among different seedling traits of wheat genotypes as affected by polyethylene glycol (PEG-6000) treatment

\begin{tabular}{|c|c|c|c|c|c|c|c|c|c|c|c|}
\hline & RG & & FG & RDW & SDW & RSDW & TDW & $\mathrm{R}: \mathrm{S}$ & ASR & SME & VI \\
\hline & & & 0.45 & 0.3 & 0.64 & -0.57 & -0.37 & -0.39 & 0.46 & -0.22 & 0.6 \\
\hline \multirow[t]{2}{*}{ RG } & & 1 & NS & NS & $* *$ & $* *$ & NS & $* *$ & $* *$ & NS & $* *$ \\
\hline & & & & 0.39 & 0.44 & -.44 & -0.25 & -0.2 & 0.22 & -0.08 & 0.78 \\
\hline \multirow[t]{2}{*}{$\mathrm{FG}$} & & & 1 & NS & NS & NS & NS & NS & NS & NS & $* *$ \\
\hline & & & & & 0.4 & -0.29 & 0.1 & 0.37 & 0.12 & 0.22 & 0.7 \\
\hline \multirow[t]{2}{*}{ RDW } & & & & 1 & NS & NS & NS & NS & NS & NS & $* *$ \\
\hline & & & & & & -0.73 & -0.38 & -0.63 & 0.54 & -0.18 & 0.81 \\
\hline \multirow[t]{2}{*}{ SDW } & & & & & 1 & $* *$ & $* *$ & $* *$ & $* *$ & NS & $* *$ \\
\hline & & & & & & & 0.88 & 0.43 & -0.64 & 0.39 & -0.65 \\
\hline \multirow[t]{2}{*}{ RSDW } & & & & & & 1 & $* *$ & NS & $* *$ & NS & $* *$ \\
\hline & & & & & & & & 0.39 & -0.56 & 0.48 & -0.27 \\
\hline \multirow[t]{2}{*}{ TDW } & & & & & & & 1 & NS & $* *$ & $* *$ & NS \\
\hline & & & & & & & & & -0.38 & 0.29 & -0.25 \\
\hline \multirow[t]{2}{*}{$\mathrm{R}: \mathrm{S}$} & & & & & & & & 1 & NS & NS & NS \\
\hline & & & & & & & & & & -0.8 & 0.42 \\
\hline \multirow[t]{2}{*}{ ASR } & & & & & & & & & 1 & $* *$ & NS \\
\hline & & & & & & & & & & & -0.06 \\
\hline SME & & & & & & & & & & 1 & NS \\
\hline VI & & & & & & & & & & & 1 \\
\hline
\end{tabular}

**Significant at $1 \%$ probability level, NS=Non-significant

RG-Rate of germination, FG-Final germination, RDW- Root dry weight, SDW-Shoot dry weight, RSDW- remaining seed dry weight, TDW-Total dry weight, R:S-Root -shoot ratio, SMR - amount of seed material respired, SME-Seed metabolic efficiency, VI-Vigour index 


\section{CONCLUSION}

Significant positive correlation exists among some important growth parameters like rate of germination (\%), final germination (\%), shoot and root dry weight with vigour index. On the basis of these physiological traits against osmotic stress, nine wheat genotypes such as BD-480, BD-498, BD-501, BD-513, BD-514, BD-519, BD-592, BD-618 and BD- 633 were selected as drought tolerant.

\section{REFERENCES}

Ahmad, S., Ahmad, R., Ashraf, M.Y., Ashraf, M., and Waraich, E.A. (2009). Sunflower (Helianthus Annuus L.) response to drought stress at germination and seedling growth stages. Pakistan Journal of Botany, 41, 647-654.

Azam, G., and Allen, R.E. (1976). Interrelationship of seedling vigor criterion of wheat under different field situations and soil water potentials. Crop Science, 16, 615-618.

Baloch, M.J., Dunwell, J., Khakwani, A.A., Dennet, M., Jatoi, W.A., and Channa, S.A. (2012). Assessment of wheat cultivars for drought tolerance via osmotic stress imposed at early seedling growth stages. Journal of Agricultural Research, 50(3), 299-310.

Bayoumi, T.Y., Manal, H.E., and Metwali, E.M. (2008). Application of physiological and biological and biochemical indices as a screening technique for drought tolerance in wheat genotypes. African Journal of Biotechnology, 7(14), 2341-2352.

Brigg, K.G. and Aytenfisu, A. (1979). The effect of seedling rate, seeding date and location on grain yield, maturity, protein percentage and protein yield of some spring wheats in central Alberia. Canadian Journal of Plant Science, 59, 1129-1146.

Dhanda, S.S., Sethi G.S., and Behl, R.K. (2004). Indices of drought tolerance in wheat genotypes at early stages of plant growth. Journal of Agronomy and Crop Science, 190, 6-12.

Galle, A., Haldimann, P., and Feller, U. (2007). Photosynthetic performance and water relations in young pubescent oak (Quercus pubescens) trees during drought stress and recovery. New Phytologist, 174,799-810.

Jiang, Y., Macdonald, S.E., and Zwiazak, J.J. (1995). Effects of cold storage and water stress on water relations and gas exchange of white spruce (Picea glauca) seedlings. Tree Physiology, 15(4), 267-273.

Krishnasamy, V., and Seshu, D.V. (1990). Germination after accelerated aging and associated characters in rice varieties. Seed Science and Technology, 18, 353-359.

Pan, X.Y., Wang, Y.F., Wang, G.X., Cao, Q.D., and Wang, J. (2002). Relationship between growth redundancy and size inequality in spring wheat population mulched with clear plastic film. Chinese Journal of Plant Ecology, 26(2), 177-184.

Rao, D.G., and Sinha, S.K. (1993). Efficiency of mobilization of seed reserves in sorghum hybrids and their parents as influnced by temperature. Seed Research, 21(2), 97-100. 
Rauf, M., Munir, M., Ul-Hassan, M., Ahmed, M., and Afzal, M. (2007). Performance of wheat genotypes under osmotic stress at germination and early seedling growth stage. African Journal of Biotechnology, 6(8), 971-975.

Roza, G. M., ShahzadJamaati-e-Somarin, K., and Zabihi-e-Mahmoodabad, R. (2010). Effects of polyethylene glycol and NaCI stress on two cultivars of wheat (Triticum durum) at germination and early seedling stages. American-Eurasian Journal of Agricultural \& Environmental Science, 9, 86-90. 
Table 1. Effect of drought stress through polyethylene glycol (PEG-6000) solution on rate of germination, final germination, dry weight of root and shoot wheat genotypes.

\begin{tabular}{|c|c|c|c|c|c|c|c|c|c|c|c|c|}
\hline \multirow[t]{2}{*}{ Genotypes } & \multicolumn{3}{|c|}{$\begin{array}{c}\text { *Rate of Germination } \\
(\%)\end{array}$} & \multicolumn{3}{|c|}{$\begin{array}{c}\text { *Final Germination } \\
(\%)\end{array}$} & \multicolumn{3}{|c|}{ Root dry weight (mg) } & \multicolumn{3}{|c|}{ Shoot dry weight $(\mathrm{mg})$} \\
\hline & Control & $\begin{array}{l}\text { PEG } \\
15 \% \\
\end{array}$ & $\begin{array}{l}\text { PEG } \\
25 \%\end{array}$ & Control & $\begin{array}{l}\text { PEG } \\
15 \%\end{array}$ & $\begin{array}{l}\text { PEG } \\
25 \%\end{array}$ & Control & \begin{tabular}{|l} 
PEG \\
$15 \%$ \\
\end{tabular} & $\begin{array}{l}\text { PEG } \\
25 \%\end{array}$ & Control & $\begin{array}{l}\text { PEG } \\
15 \%\end{array}$ & $\begin{array}{l}\text { PEG } \\
25 \%\end{array}$ \\
\hline BD-466 & 49 & 22 & 10 & 82 & 71 & 24 & 3.7 & 3.6 & 3.3 & 6.7 & 4.6 & 4.3 \\
\hline BD-470 & 60 & 38 & 5 & 85 & 67 & 36 & 5.0 & 5.1 & 5.6 & 9.8 & 5.1 & 4.1 \\
\hline BD-473 & 82 & 42 & 7 & 92 & 74 & 63 & 3.6 & 4.5 & 3.7 & 7.9 & 5.0 & 3.9 \\
\hline BD-476 & 64 & 36 & 11 & 90 & 69 & 42 & 4.5 & 4.1 & 2.6 & 8.4 & 5.5 & 4.5 \\
\hline BD-479 & 75 & 35 & 7 & 93 & 72 & 41 & 3.9 & 4.3 & 3.3 & 8.1 & 4.9 & 4.2 \\
\hline BD-480 & 79 & 43 & 10 & 95 & 84 & 76 & 4.2 & 4.5 & 3.5 & 8.4 & 5.1 & 4.2 \\
\hline BD-481 & 68 & 44 & 10 & 88 & 69 & 59 & 3.4 & 4.2 & 3.7 & 7.2 & 5.5 & 4.7 \\
\hline BD-483 & 83 & 54 & 40 & 78 & 58 & 54 & 4.1 & 3.7 & 4.2 & 7.9 & 7.0 & 5.3 \\
\hline BD-487 & 56 & 29 & 10 & 88 & 77 & 38 & 4.0 & 4.6 & 3.0 & 8.2 & 5.5 & 4.0 \\
\hline BD-488 & 64 & 46 & 19 & 75 & 56 & 38 & 4.4 & 3.7 & 2.8 & 9.3 & 6.2 & 4.6 \\
\hline BD-489 & 76 & 43 & 11 & 95 & 75 & 62 & 4.1 & 5.3 & 4.0 & 8.4 & 5.5 & 3.9 \\
\hline BD-491 & 72 & 45 & 20 & 88 & 71 & 60 & 4.0 & 4.4 & 4.7 & 9.1 & 6.3 & 5.8 \\
\hline BD-493 & 94 & 83 & 20 & 87 & 65 & 63 & 5.1 & 6.4 & 0.5 & 9.9 & 7.8 & 8.3 \\
\hline BD-496 & 97 & 92 & 17 & 100 & 82 & 69 & 7.1 & 6.0 & 7.0 & 10.6 & 8.6 & 8.4 \\
\hline BD-497 & 98 & 91 & 8 & 85 & 72 & 64 & 5.7 & 6.6 & 7.1 & 11.6 & 7.0 & 8.6 \\
\hline BD-498 & 93 & 87 & 13 & 90 & 81 & 68 & 5.1 & 4.8 & 5.6 & 7.9 & 6.9 & 6.7 \\
\hline BD-499 & 98 & 88 & 16 & 98 & 83 & 70 & 5.5 & 6.6 & 8.2 & 10.6 & 9.5 & 9.5 \\
\hline BD-500 & 94 & 90 & 2 & 88 & 74 & 66 & 6.3 & 5.1 & 7.2 & 9.7 & 5.8 & 7.8 \\
\hline BD-501 & 93 & 91 & 15 & 95 & 81 & 70 & 6.5 & 7.1 & 7.2 & 9.2 & 9.5 & 7.8 \\
\hline BD-505 & 94 & 90 & 22 & 88 & 75 & 69 & 6.2 & 6.7 & 6.9 & 10.0 & 8.0 & 7.4 \\
\hline BD-507 & 96 & 76 & 11 & 88 & 72 & 70 & 5.9 & 5.3 & 6.9 & 9.9 & 5.8 & 8.8 \\
\hline BD-508 & 93 & 95 & 17 & 98 & 83 & 69 & 6.8 & 5.6 & 6.4 & 8.8 & 8.2 & 7.6 \\
\hline BD-509 & 86 & 77 & 4 & 98 & 57 & 56 & 5.6 & 5.6 & 6.1 & 9.5 & 7.8 & 7.6 \\
\hline BD-510 & 98 & 91 & 13 & 100 & 87 & 72 & 5.5 & 6.0 & 6.5 & 11.9 & 6.5 & 8.4 \\
\hline BD-511 & 73 & 70 & 19 & 87 & 72 & 68 & 7.1 & 5.7 & 6.9 & 9.1 & 5.7 & 6.2 \\
\hline BD-512 & 94 & 88 & 8 & 83 & 69 & 65 & 5.8 & 5.7 & 6.9 & 9.5 & 5.0 & 5.5 \\
\hline BD-513 & 93 & 91 & 16 & 75 & 63 & 65 & 6.1 & 7.8 & 6.7 & 7.0 & 6.2 & 5.7 \\
\hline BD-514 & 98 & 97 & 39 & 97 & 78 & 63 & 3.9 & 5.2 & 6.5 & 5.1 & 7.0 & 5.7 \\
\hline BD-516 & 97 & 90 & 20 & 98 & 81 & 71 & 5.2 & 5.1 & 7.0 & 9.9 & 5.5 & 5.7 \\
\hline BD-517 & 98 & 97 & 2 & 80 & 80 & 62 & 4.1 & 4.8 & 6.8 & 9.1 & 5.0 & 5.8 \\
\hline BD-519 & 98 & 95 & 19 & 95 & 81 & 67 & 3.0 & 5.1 & 7.1 & 7.0 & 5.0 & 5.4 \\
\hline BD-522 & 69 & 68 & 67 & 85 & 74 & 61 & 4.4 & 5.5 & 7.9 & 10.0 & 6.0 & 6.9 \\
\hline BD-524 & 97 & 95 & 18 & 95 & 84 & 68 & 4.7 & 4.4 & 7.0 & 7.7 & 4.8 & 6.8 \\
\hline BD-525 & 86 & 73 & 5 & 95 & 71 & 66 & 2.4 & 4.3 & 4.8 & 5.0 & 4.2 & 4.9 \\
\hline BD-527 & 98 & 81 & 2 & 92 & 75 & 62 & 2.8 & 3.8 & 4.2 & 6.1 & 4.1 & 4.2 \\
\hline BD-529 & 85 & 78 & 7 & 98 & 81 & 71 & 6.2 & 3.8 & 6.3 & 7.1 & 3.6 & 4.9 \\
\hline
\end{tabular}




\begin{tabular}{|c|c|c|c|c|c|c|c|c|c|c|c|c|}
\hline \multirow[t]{2}{*}{ Genotypes } & \multicolumn{3}{|c|}{$\begin{array}{c}* \text { Rate of Germination } \\
(\%)\end{array}$} & \multicolumn{3}{|c|}{$\begin{array}{c}\text { *Final Germination } \\
(\%)\end{array}$} & \multicolumn{3}{|c|}{ Root dry weight (mg) } & \multicolumn{3}{|c|}{ Shoot dry weight $(\mathrm{mg})$} \\
\hline & Control & $\begin{array}{l}\text { PEG } \\
15 \% \\
\end{array}$ & $\begin{array}{l}\text { PEG } \\
25 \% \\
\end{array}$ & Control & $\begin{array}{l}\text { PEG } \\
15 \% \\
\end{array}$ & $\begin{array}{l}\text { PEG } \\
25 \% \\
\end{array}$ & Control & $\begin{array}{l}\text { PEG } \\
15 \% \\
\end{array}$ & $\begin{array}{l}\text { PEG } \\
25 \% \\
\end{array}$ & Control & $\begin{array}{l}\text { PEG } \\
15 \% \\
\end{array}$ & $\begin{array}{l}\text { PEG } \\
25 \% \\
\end{array}$ \\
\hline BD-531 & 98 & 97 & 6 & 97 & 81 & 71 & 6.7 & 6.8 & 3.8 & 8.7 & 5.4 & 3.3 \\
\hline BD-533 & 98 & 87 & 22 & 98 & 81 & 63 & 3.8 & 6.5 & 4.9 & 8.7 & 4.7 & 4.8 \\
\hline BD-534 & 98 & 51 & 40 & 98 & 78 & 57 & 6.8 & 6.0 & 4.3 & 7.9 & 5.0 & 4.6 \\
\hline BD-535 & 93 & 48 & 33 & 87 & 69 & 41 & 6.3 & 5.2 & 5.5 & 9.4 & 4.1 & 4.3 \\
\hline BD-536 & 98 & 54 & 22 & 98 & 87 & 69 & 9.0 & 4.3 & 6.5 & 8.8 & 5.7 & 4.6 \\
\hline BD-537 & 90 & 34 & 25 & 95 & 83 & 54 & 7.8 & 5.1 & 5.9 & 8.0 & 3.2 & 4.5 \\
\hline BD-539 & 90 & 25 & 30 & 88 & 78 & 36 & 8.8 & 5.0 & 3.2 & 10.0 & 4.2 & 3.1 \\
\hline BD-540 & 92 & 31 & 20 & 88 & 71 & 21 & 6.8 & 5.2 & 6.8 & 9.2 & 5.2 & 8.6 \\
\hline BD-541 & 98 & 27 & 33 & 98 & 81 & 51 & 7.6 & 5.3 & 3.9 & 9.5 & 4.5 & 4.9 \\
\hline BD-542 & 96 & 57 & 20 & 83 & 69 & 60 & 5.3 & 6.2 & 2.3 & 7.4 & 4.6 & 3.3 \\
\hline BD-545 & 73 & 32 & 18 & 93 & 81 & 60 & 8.7 & 5.3 & 4.9 & 9.4 & 3.6 & 4.5 \\
\hline BD-546 & 63 & 19 & 10 & 47 & 27 & 8 & 6.3 & 3.0 & 1.9 & 9.2 & 2.7 & 2.3 \\
\hline BD-547 & 97 & 43 & 11 & 98 & 86 & 68 & 7.1 & 5.0 & 4.5 & 8.7 & 4.3 & 5.3 \\
\hline BD-551 & 35 & 24 & 20 & 85 & 39 & 3 & 4.2 & 2.9 & 5.0 & 6.8 & 3.5 & 1.8 \\
\hline BD-552 & 82 & 28 & 9 & 90 & 68 & 66 & 5.7 & 6.2 & 4.1 & 8.3 & 6.1 & 4.0 \\
\hline BD-560 & 76 & 33 & 13 & 95 & 83 & 56 & 6.3 & 5.6 & 4.0 & 7.8 & 5.3 & 4.2 \\
\hline BD-562 & 95 & 54 & 21 & 100 & 84 & 72 & 7.1 & 6.7 & 5.0 & 9.5 & 5.7 & 4.8 \\
\hline BD-563 & 88 & 48 & 11 & 98 & 86 & 71 & 7.6 & 7.1 & 5.5 & 10.1 & 6.1 & 4.1 \\
\hline BD-567 & 84 & 54 & 22 & 98 & 86 & 74 & 6.7 & 5.3 & 3.9 & 6.9 & 5.4 & 4.6 \\
\hline BD-568 & 98 & 27 & 14 & 100 & 74 & 66 & 7.1 & 5.7 & 4.4 & 8.3 & 4.1 & 4.5 \\
\hline BD-569 & 85 & 20 & 16 & 100 & 89 & 59 & 7.1 & 6.0 & 4.8 & 9.0 & 5.6 & 4.5 \\
\hline BD-570 & 83 & 41 & 10 & 90 & 84 & 32 & 6.0 & 5.0 & 4.1 & 8.7 & 4.5 & 3.4 \\
\hline BD-574 & 85 & 40 & 3 & 97 & 80 & 32 & 5.6 & 6.6 & 4.1 & 7.7 & 5.4 & 4.5 \\
\hline BD-576 & 96 & 46 & 25 & 97 & 86 & 60 & 5.6 & 6.0 & 3.9 & 8.5 & 6.2 & 4.6 \\
\hline BD-579 & 95 & 39 & 8 & 98 & 86 & 51 & 8.1 & 6.7 & 3.3 & 9.2 & 6.4 & 4.4 \\
\hline BD-581 & 89 & 47 & 10 & 90 & 78 & 59 & 5.7 & 5.7 & 4.9 & 9.2 & 4.9 & 3.5 \\
\hline BD-582 & 95 & 58 & 32 & 93 & 77 & 68 & 8.0 & 8.0 & 6.5 & 9.5 & 6.2 & 4.8 \\
\hline BD-583 & 95 & 47 & 22 & 98 & 83 & 68 & 8.1 & 6.1 & 5.1 & 8.5 & 6.5 & 4.8 \\
\hline BD-584 & 97 & 42 & 27 & 97 & 78 & 69 & 6.1 & 6.0 & 3.6 & 9.1 & 6.8 & 4.0 \\
\hline BD-590 & 93 & 12 & 28 & 97 & 80 & 70 & 5.7 & 3.7 & 3.6 & 7.7 & 4.1 & 3.4 \\
\hline BD-592 & 94 & 98 & 19 & 85 & 84 & 74 & 6.5 & 8.2 & 7.1 & 9.4 & 7.7 & 6.1 \\
\hline BD-593 & 97 & 63 & 8 & 98 & 81 & 60 & 4.7 & 5.8 & 5.6 & 7.5 & 6.1 & 5.5 \\
\hline BD-598 & 97 & 93 & 7 & 100 & 87 & 60 & 5.8 & 8.0 & 6.5 & 9.8 & 6.0 & 4.4 \\
\hline BD-599 & 97 & 95 & 7 & 97 & 84 & 66 & 7.2 & 8.7 & 5.9 & 8.6 & 7.3 & 5.5 \\
\hline BD-600 & 97 & 88 & 7 & 97 & 87 & 72 & 7.4 & 8.5 & 5.7 & 10.5 & 7.8 & 6.2 \\
\hline BD-601 & 98 & 93 & 7 & 95 & 84 & 56 & 6.6 & 8.3 & 6.0 & 10.2 & 7.0 & 5.3 \\
\hline BD-602 & 96 & 98 & 20 & 95 & 80 & 72 & 5.9 & 6.9 & 5.2 & 9.3 & 8.2 & 6.6 \\
\hline BD-603 & 98 & 98 & 18 & 95 & 81 & 67 & 7.2 & 8.0 & 6.2 & 9.5 & 8.2 & 7.1 \\
\hline BD-604 & 98 & 93 & 27 & 93 & 82 & 71 & 4.4 & 6.3 & 4.7 & 10.0 & 7.3 & 6.1 \\
\hline
\end{tabular}




\begin{tabular}{|c|c|c|c|c|c|c|c|c|c|c|c|c|}
\hline \multirow[t]{2}{*}{ Genotypes } & \multicolumn{3}{|c|}{$\begin{array}{c}* \text { Rate of Germination } \\
(\%)\end{array}$} & \multicolumn{3}{|c|}{$\begin{array}{c}\text { *Final Germination } \\
(\%)\end{array}$} & \multicolumn{3}{|c|}{ Root dry weight $(\mathrm{mg})$} & \multicolumn{3}{|c|}{ Shoot dry weight $(\mathrm{mg})$} \\
\hline & Control & $\begin{array}{l}\text { PEG } \\
15 \%\end{array}$ & $\begin{array}{l}\text { PEG } \\
25 \%\end{array}$ & Control & \begin{tabular}{|l|} 
PEG \\
$15 \%$ \\
\end{tabular} & \begin{tabular}{|l|} 
PEG \\
$25 \%$
\end{tabular} & Control & \begin{tabular}{|l|} 
PEG \\
$15 \%$
\end{tabular} & $\begin{array}{l}\text { PEG } \\
25 \%\end{array}$ & Control & $\begin{array}{l}\text { PEG } \\
15 \%\end{array}$ & $\begin{array}{l}\text { PEG } \\
25 \%\end{array}$ \\
\hline BD-605 & 95 & 98 & 10 & 98 & 87 & 74 & 6.3 & 7.0 & 5.3 & 10.3 & 6.9 & 5.6 \\
\hline BD-607 & 92 & 87 & 22 & 85 & 75 & 60 & 5.5 & 7.1 & 4.7 & 10.0 & 6.7 & 5.5 \\
\hline BD-608 & 97 & 88 & 75 & 93 & 77 & 62 & 6.4 & 7.8 & 5.8 & 9.8 & 7.8 & 5.8 \\
\hline BD-610 & 40 & 10 & 10 & 85 & 33 & 11 & 4.6 & 4.5 & 3.6 & 9.0 & 7.0 & 4.8 \\
\hline BD-611 & 95 & 87 & 5 & 92 & 82 & 65 & 4.9 & 7.0 & 4.1 & 9.6 & 6.5 & 4.9 \\
\hline BD-612 & 97 & 90 & 65 & 93 & 83 & 67 & 5.9 & 6.8 & 8.1 & 9.5 & 6.7 & 5.7 \\
\hline BD-613 & 96 & 95 & 47 & 92 & 79 & 65 & 6.8 & 6.8 & 6.4 & 10.2 & 6.5 & 5.3 \\
\hline BD-614 & 96 & 88 & 25 & 92 & 81 & 60 & 5.9 & 7.0 & 4.1 & 8.4 & 8.2 & 7.1 \\
\hline BD-616 & 97 & 91 & 40 & 95 & 84 & 66 & 5.7 & 5.7 & 4.8 & 7.8 & 5.8 & 4.6 \\
\hline BD-617 & 95 & 90 & 55 & 95 & 75 & 59 & 5.8 & 7.5 & 5.9 & 9.3 & 7.6 & 4.8 \\
\hline BD-618 & 93 & 87 & 62 & 80 & 66 & 56 & 3.6 & 7.6 & 6.7 & 8.4 & 7.3 & 6.4 \\
\hline BD-622 & 97 & 93 & 64 & 98 & 86 & 77 & 4.6 & 5.7 & 2.2 & 8.7 & 7.3 & 5.6 \\
\hline BD-628 & 94 & 92 & 43 & 90 & 72 & 51 & 5.2 & 5.3 & 4.9 & 8.0 & 6.2 & 4.2 \\
\hline BD-629 & 98 & 86 & 16 & 87 & 71 & 51 & 3.6 & 7.0 & 6.2 & 9.0 & 5.8 & 4.4 \\
\hline BD-632 & 93 & 89 & 49 & 97 & 83 & 66 & 4.9 & 7.0 & 5.9 & 8.6 & 5.5 & 4.2 \\
\hline BD-633 & 95 & 91 & 44 & 90 & 74 & 68 & 5.3 & 7.3 & 6.2 & 8.3 & 9.0 & 6.1 \\
\hline BD-634 & 98 & 93 & 41 & 92 & 77 & 66 & 5.5 & 7.1 & 7.3 & 10.2 & 7.6 & 5.6 \\
\hline BD-635 & 93 & 93 & 31 & 100 & 77 & 69 & 5.5 & 5.8 & 5.1 & 10.5 & 7.3 & 5.0 \\
\hline BD-636 & 96 & 90 & 52 & 92 & 80 & 71 & 5.2 & 5.0 & 5.7 & 8.4 & 5.8 & 4.6 \\
\hline BD-638 & 95 & 91 & 11 & 93 & 83 & 48 & 5.6 & 4.0 & 5.3 & 10.8 & 7.7 & 4.9 \\
\hline BD-641 & 92 & 82 & 28 & 80 & 71 & 59 & 4.1 & 5.1 & 3.4 & 8.0 & 5.1 & 4.0 \\
\hline BD-642 & 98 & 93 & 64 & 77 & 71 & 59 & 4.1 & 5.7 & 3.4 & 8.2 & 5.5 & 5.5 \\
\hline BD-643 & 93 & 91 & 62 & 100 & 81 & 54 & 5.8 & 6.0 & 4.4 & 9.5 & 6.2 & 5.6 \\
\hline BD-644 & 98 & 95 & 65 & 85 & 71 & 51 & 4.2 & 5.0 & 4.3 & 9.0 & 6.1 & 4.3 \\
\hline BD-645 & 95 & 93 & 69 & 87 & 74 & 66 & 5.1 & 5.7 & 4.8 & 9.0 & 6.1 & 5.3 \\
\hline $\operatorname{LSD}(0.05)$ & & 14.36 & & & 9.93 & & & 1.47 & & & 1.46 & \\
\hline CV (\%) & & 14.03 & & & 8.29 & & & 13.49 & & & 10.99 & \\
\hline
\end{tabular}

* Percentage data were transformed into arcsine value for analysis 
Table 2. Effect of drought stress through polyethylene glycol (PEG-6000) solution on remaining seed dry weight, Root - Shoot ratio, amount of respiration and seed metabolic efficiency wheat genotypes.

\begin{tabular}{|c|c|c|c|c|c|c|c|c|c|c|c|c|}
\hline \multirow[t]{2}{*}{ Genotypes } & \multicolumn{3}{|c|}{$\begin{array}{l}\text { Remaining seed dry } \\
\text { weight (mg) }\end{array}$} & \multicolumn{3}{|c|}{ Root:Shoot } & \multicolumn{3}{|c|}{$\begin{array}{l}\text { Amount of } \\
\text { respiration }\end{array}$} & \multicolumn{3}{|c|}{$\begin{array}{l}\text { Seed metabolic } \\
\text { efficiency }(\mathrm{g} / \mathrm{g})\end{array}$} \\
\hline & $\begin{array}{c}\text { Contro } \\
1\end{array}$ & $\begin{array}{l}\text { PEG } \\
15 \%\end{array}$ & $\begin{array}{l}\text { PEG } \\
25 \%\end{array}$ & $\begin{array}{c}\text { Contro } \\
1\end{array}$ & $\begin{array}{l}\text { PEG } \\
15 \%\end{array}$ & $\begin{array}{l}\text { PEG } \\
25 \%\end{array}$ & Control & $\begin{array}{l}\text { PEG } \\
15 \%\end{array}$ & $\begin{array}{l}\text { PEG } \\
25 \%\end{array}$ & Control & $\begin{array}{l}\text { PEG } \\
15 \%\end{array}$ & $\begin{array}{l}\text { PEG } \\
25 \%\end{array}$ \\
\hline BD-466 & 4.4 & 10.0 & 12.2 & 0.6 & 0.8 & 0.8 & 6.0 & 2.7 & 3.0 & 1.7 & 3.0 & 2.5 \\
\hline BD-470 & 9.3 & 19.9 & 27.7 & 0.5 & 1.0 & 1.4 & 14.0 & 8.0 & 2.8 & 1.1 & 1.3 & 3.5 \\
\hline BD-473 & 7.2 & 15.1 & 18.3 & 0.5 & 0.9 & 0.9 & 13.1 & 7.1 & 5.8 & 0.9 & 1.3 & 1.3 \\
\hline BD-476 & 7.3 & 15.9 & 23.4 & 0.5 & 0.7 & 0.6 & 15.8 & 10.6 & 5.5 & 0.8 & 0.9 & 1.3 \\
\hline BD-479 & 7.2 & 17.5 & 21.5 & 0.5 & 0.9 & 0.8 & 17.6 & 10.1 & 7.9 & 0.7 & 0.9 & 0.9 \\
\hline BD-480 & 5.7 & 13.3 & 18.5 & 0.5 & 0.9 & 0.8 & 11.5 & 6.9 & 3.7 & 1.1 & 1.4 & 2.1 \\
\hline BD-481 & 6.9 & 13.3 & 17.4 & 0.5 & 0.8 & 0.8 & 15.1 & 9.5 & 6.7 & 0.7 & 1.0 & 1.3 \\
\hline BD-483 & 6.4 & 16.3 & 18.8 & 0.5 & 0.5 & 0.8 & 16.6 & 7.9 & 6.6 & 0.7 & 1.4 & 1.4 \\
\hline BD-487 & 5.7 & 14.0 & 18.4 & 0.5 & 0.8 & 0.8 & 12.2 & 6.1 & 4.9 & 1.0 & 1.6 & 1.4 \\
\hline BD-488 & 8.5 & 22.1 & 22.8 & 0.5 & 0.6 & 0.6 & 18.0 & 8.2 & 10.0 & 0.8 & 1.2 & 0.7 \\
\hline BD-489 & 5.1 & 15.3 & 21.2 & 0.5 & 1.0 & 1.0 & 15.0 & 6.5 & 3.6 & 0.8 & 1.6 & 2.2 \\
\hline BD-491 & 6.9 & 16.2 & 19.0 & 0.4 & 0.7 & 0.8 & 13.0 & 6.2 & 3.6 & 1.0 & 1.7 & 2.9 \\
\hline BD-493 & 4.0 & 7.7 & 6.2 & 0.5 & 0.8 & 0.1 & 12.2 & 9.3 & 6.2 & 1.2 & 1.5 & 1.4 \\
\hline BD-496 & 3.2 & 9.3 & 8.0 & 0.7 & 0.7 & 0.8 & 9.4 & 6.4 & 6.8 & 1.9 & 2.3 & 2.3 \\
\hline BD-497 & 4.1 & 9.7 & 7.8 & 0.5 & 0.9 & 0.8 & 9.1 & 7.2 & 6.9 & 1.9 & 1.9 & 2.3 \\
\hline BD-498 & 6.3 & 7.2 & 6.6 & 0.7 & 0.7 & 0.8 & 6.4 & 6.8 & 6.7 & 2.0 & 1.7 & 1.8 \\
\hline BD-499 & 8.9 & 11.5 & 10.7 & 0.5 & 0.7 & 0.9 & 14.2 & 11.7 & 10.8 & 1.1 & 1.4 & 1.6 \\
\hline BD-500 & 6.1 & 14.5 & 12.0 & 0.7 & 0.9 & 0.9 & 6.8 & 3.5 & 3.9 & 2.4 & 3.1 & 3.8 \\
\hline BD-501 & 6.8 & 9.4 & 10.2 & 0.7 & 0.8 & 0.9 & 14.5 & 10.9 & 11.8 & 1.1 & 1.5 & 1.3 \\
\hline BD-505 & 6.2 & 9.2 & 7.8 & 0.6 & 0.8 & 0.9 & 12.1 & 10.7 & 12.6 & 1.3 & 1.4 & 1.1 \\
\hline BD-507 & 6.1 & 13.0 & 10.4 & 0.6 & 0.9 & 0.8 & 11.1 & 8.9 & 6.9 & 1.4 & 1.3 & 2.3 \\
\hline BD-508 & 7.5 & 13.4 & 10.9 & 0.8 & 0.7 & 0.9 & 13.5 & 9.3 & 11.6 & 1.2 & 1.5 & 1.2 \\
\hline BD-509 & 8.2 & 12.5 & 12.5 & 0.6 & 0.7 & 0.8 & 10.8 & 8.1 & 7.8 & 1.4 & 1.7 & 1.8 \\
\hline BD-510 & 7.8 & 14.5 & 12.1 & 0.5 & 0.9 & 0.8 & 9.3 & 7.5 & 7.6 & 1.9 & 1.7 & 2.0 \\
\hline BD-511 & 6.8 & 17.4 & 16.3 & 0.8 & 1.0 & 1.1 & 8.7 & 2.9 & 3.5 & 1.9 & 3.9 & 3.7 \\
\hline BD-512 & 9.2 & 21.1 & 15.5 & 0.6 & 1.1 & 1.3 & 12.7 & 5.4 & 9.3 & 1.2 & 2.0 & 1.3 \\
\hline BD-513 & 14.9 & 17.4 & 16.9 & 0.4 & 1.3 & 1.2 & 9.4 & 4.1 & 5.2 & 1.1 & 3.4 & 2.4 \\
\hline BD-514 & 10.4 & 18.2 & 14.6 & 0.6 & 0.7 & 1.2 & 10.5 & 4.5 & 7.1 & 0.9 & 2.7 & 1.7 \\
\hline BD-516 & 10.8 & 23.7 & 16.2 & 0.5 & 0.9 & 1.2 & 11.2 & 2.8 & 8.2 & 1.4 & 3.8 & 1.6 \\
\hline BD-517 & 14.2 & 23.5 & 20.6 & 0.5 & 1.0 & 1.2 & 9.5 & 3.6 & 3.7 & 1.4 & 2.7 & 3.4 \\
\hline BD-519 & 13.1 & 17.0 & 13.9 & 0.4 & 1.0 & 1.3 & 6.4 & 4.3 & 4.0 & 1.6 & 2.3 & 3.1 \\
\hline BD-522 & 20.3 & 29.2 & 21.8 & 0.4 & 0.9 & 1.2 & 8.3 & 3.3 & 6.4 & 1.7 & 3.4 & 2.3 \\
\hline BD-524 & 8.6 & 16.6 & 16.3 & 0.6 & 0.9 & 1.0 & 12.4 & 7.6 & 4.2 & 1.0 & 1.2 & 3.3 \\
\hline BD-525 & 10.6 & 12.8 & 10.0 & 0.5 & 1.0 & 1.0 & 5.6 & 2.3 & 4.0 & 1.3 & 3.8 & 2.4 \\
\hline BD-527 & 13.5 & 19.8 & 18.6 & 0.5 & 0.9 & 1.0 & 10.6 & 5.4 & 6.1 & 0.9 & 1.5 & 1.4 \\
\hline BD-529 & 9.7 & 16.9 & 13.4 & 0.9 & 1.1 & 1.3 & 11.1 & 9.7 & 9.5 & 1.2 & 0.8 & 1.2 \\
\hline
\end{tabular}




\begin{tabular}{|c|c|c|c|c|c|c|c|c|c|c|c|c|}
\hline \multirow[t]{2}{*}{ Genotypes } & \multicolumn{3}{|c|}{$\begin{array}{l}\text { Remaining seed dry } \\
\text { weight (mg) }\end{array}$} & \multicolumn{3}{|c|}{ Root:Shoot } & \multicolumn{3}{|c|}{$\begin{array}{l}\text { Amount of } \\
\text { respiration }\end{array}$} & \multicolumn{3}{|c|}{$\begin{array}{l}\text { Seed metabolic } \\
\text { efficiency }(\mathrm{g} / \mathrm{g})\end{array}$} \\
\hline & $\begin{array}{c}\text { Contro } \\
1\end{array}$ & $\begin{array}{l}\text { PEG } \\
15 \%\end{array}$ & $\begin{array}{l}\text { PEG } \\
25 \%\end{array}$ & $\begin{array}{c}\text { Contro } \\
1\end{array}$ & $\begin{array}{l}\text { PEG } \\
15 \%\end{array}$ & $\begin{array}{l}\text { PEG } \\
25 \%\end{array}$ & Control & $\begin{array}{l}\text { PEG } \\
15 \%\end{array}$ & $\begin{array}{l}\text { PEG } \\
25 \%\end{array}$ & Control & \begin{tabular}{|l|} 
PEG \\
$15 \%$
\end{tabular} & $\begin{array}{l}\text { PEG } \\
25 \%\end{array}$ \\
\hline BD-531 & 4.2 & 12.1 & 19.5 & 0.8 & 1.3 & 1.1 & 14.6 & 10.0 & 7.7 & 1.1 & 1.2 & 0.9 \\
\hline BD-533 & 5.3 & 12.7 & 16.4 & 0.4 & 1.4 & 1.0 & 13.6 & 7.6 & 5.4 & 0.9 & 1.5 & 1.8 \\
\hline BD-534 & 5.6 & 14.0 & 18.6 & 0.9 & 1.2 & 0.9 & 11.9 & 7.2 & 4.6 & 1.2 & 1.5 & 1.9 \\
\hline BD-535 & 3.9 & 12.9 & 17.0 & 0.7 & 1.1 & 1.1 & 15.2 & 12.4 & 7.9 & 1.0 & 0.8 & 2.5 \\
\hline BD-536 & 4.0 & 11.8 & 15.5 & 1.0 & 0.8 & 1.4 & 14.9 & 14.9 & 10.1 & 1.2 & 0.7 & 1.1 \\
\hline BD-537 & 4.7 & 12.4 & 19.1 & 1.0 & 1.6 & 1.3 & 17.6 & 17.3 & 8.5 & 0.9 & 0.5 & 1.2 \\
\hline BD-539 & 5.1 & 16.6 & 25.0 & 0.9 & 1.2 & 1.0 & 14.6 & 12.7 & 7.2 & 1.3 & 0.7 & 0.9 \\
\hline BD-540 & 5.9 & 15.1 & 15.3 & 0.7 & 1.0 & 1.2 & 16.8 & 13.2 & 8.1 & 1.0 & 0.8 & 3.2 \\
\hline BD-541 & 5.1 & 15.3 & 21.4 & 0.8 & 1.2 & 0.8 & 13.8 & 10.7 & 5.7 & 1.2 & 0.9 & 1.6 \\
\hline BD-542 & 4.0 & 14.7 & 19.4 & 0.7 & 1.4 & 0.7 & 14.8 & 6.0 & 6.6 & 0.9 & 1.8 & 0.9 \\
\hline BD-545 & 4.3 & 15.0 & 22.0 & 0.9 & 1.5 & 1.1 & 15.7 & 14.1 & 6.6 & 1.2 & 0.6 & 1.4 \\
\hline BD-546 & 8.0 & 21.7 & 16.5 & 0.7 & 1.1 & 0.8 & 16.6 & 12.6 & 19.3 & 0.9 & 0.5 & 0.2 \\
\hline BD-547 & 5.1 & 17.1 & 18.6 & 0.8 & 1.2 & 0.8 & 14.0 & 8.5 & 6.6 & 1.1 & 1.1 & 1.5 \\
\hline BD-551 & 7.3 & 10.1 & 10.8 & 0.6 & 0.8 & 2.7 & 7.8 & 9.7 & 9.1 & 1.4 & 0.7 & 0.8 \\
\hline BD-552 & 4.6 & 13.9 & 17.7 & 0.7 & 1.0 & 1.0 & 14.4 & 6.7 & 7.2 & 1.0 & 1.8 & 1.1 \\
\hline BD-560 & 5.2 & 14.8 & 20.5 & 0.8 & 1.0 & 0.9 & 13.0 & 6.5 & 3.6 & 1.1 & 1.7 & 2.3 \\
\hline BD-562 & 5.0 & 15.4 & 19.4 & 0.8 & 1.2 & 1.1 & 14.7 & 8.5 & 7.1 & 1.1 & 1.5 & 1.4 \\
\hline BD-563 & 7.4 & 22.0 & 29.2 & 0.8 & 1.2 & 1.3 & 16.8 & 8.7 & 5.2 & 1.1 & 1.5 & 1.8 \\
\hline BD-567 & 5.1 & 17.1 & 20.9 & 1.0 & 1.0 & 0.9 & 14.8 & 5.6 & 4.0 & 0.9 & 1.9 & 2.1 \\
\hline BD-568 & 3.5 & 15.6 & 17.6 & 0.9 & 1.4 & 1.0 & 11.8 & 5.4 & 4.2 & 1.3 & 1.8 & 2.1 \\
\hline BD-569 & 5.0 & 19.1 & 24.3 & 0.8 & 1.1 & 1.1 & 17.2 & 7.6 & 4.6 & 0.9 & 1.5 & 2.0 \\
\hline BD-570 & 4.1 & 15.9 & 18.8 & 0.7 & 1.1 & 1.2 & 10.3 & 3.7 & 2.9 & 1.4 & 2.6 & 2.5 \\
\hline BD-574 & 4.0 & 12.9 & 17.0 & 0.7 & 1.2 & 0.9 & 13.4 & 5.8 & 5.1 & 1.0 & 2.1 & 1.7 \\
\hline BD-576 & 4.6 & 13.3 & 18.2 & 0.7 & 1.0 & 0.8 & 11.8 & 5.0 & 3.7 & 1.2 & 2.5 & 2.3 \\
\hline BD-579 & 5.4 & 15.3 & 24.4 & 0.9 & 1.0 & 1.0 & 15.7 & 10.0 & 6.3 & 1.1 & 1.3 & 2.8 \\
\hline BD-581 & 4.9 & 14.9 & 20.5 & 0.6 & 1.2 & 1.4 & 14.1 & 8.4 & 5.0 & 1.1 & 1.3 & 1.7 \\
\hline BD-582 & 4.2 & 15.3 & 21.3 & 0.8 & 1.3 & 1.4 & 16.0 & 8.3 & 5.0 & 1.1 & 1.7 & 2.3 \\
\hline BD-583 & 6.1 & 16.7 & 25.1 & 1.0 & 0.9 & 1.1 & 15.6 & 9.1 & 3.2 & 1.1 & 1.4 & 3.1 \\
\hline BD-584 & 4.8 & 13.6 & 20.0 & 0.7 & 0.9 & 0.9 & 15.2 & 8.7 & 7.5 & 1.0 & 1.5 & 1.0 \\
\hline BD-590 & 3.3 & 15.1 & 16.2 & 0.7 & 0.9 & 1.1 & 12.2 & 6.1 & 5.7 & 1.1 & 1.3 & 1.3 \\
\hline BD-592 & 5.4 & 8.3 & 13.3 & 0.7 & 1.1 & 1.2 & 8.2 & 5.4 & 4.1 & 1.9 & 3.0 & 3.3 \\
\hline BD-593 & 6.6 & 8.3 & 13.8 & 0.6 & 0.9 & 1.0 & 8.5 & 7.2 & 3.3 & 1.4 & 1.7 & 3.4 \\
\hline BD-598 & 5.4 & 12.1 & 16.0 & 0.6 & 1.3 & 1.5 & 9.7 & 4.5 & 3.8 & 1.6 & 3.1 & 2.9 \\
\hline BD-599 & 6.5 & 12.9 & 19.9 & 0.9 & 1.2 & 1.1 & 12.1 & 5.5 & 3.1 & 1.3 & 2.9 & 3.7 \\
\hline BD-600 & 5.2 & 14.2 & 19.5 & 0.7 & 1.1 & 0.9 & 15.1 & 7.7 & 6.8 & 1.2 & 2.1 & 1.8 \\
\hline BD-601 & 5.7 & 16.2 & 20.6 & 0.7 & 1.2 & 1.1 & 11.4 & 4.4 & 4.0 & 1.5 & 3.5 & 2.9 \\
\hline BD-602 & 5.1 & 8.9 & 17.7 & 0.6 & 0.8 & 0.8 & 12.7 & 9.0 & 3.6 & 1.2 & 1.7 & 3.3 \\
\hline BD-603 & 7.1 & 13.6 & 16.7 & 0.8 & 1.0 & 0.9 & 10.7 & 4.5 & 4.4 & 1.6 & 3.6 & 3.1 \\
\hline BD-604 & 5.5 & 12.3 & 15.6 & 0.4 & 0.9 & 0.8 & 8.5 & 4.4 & 3.0 & 1.7 & 3.1 & 3.6 \\
\hline
\end{tabular}




\begin{tabular}{|c|c|c|c|c|c|c|c|c|c|c|c|c|}
\hline \multirow[t]{2}{*}{ Genotypes } & \multicolumn{3}{|c|}{$\begin{array}{l}\text { Remaining seed dry } \\
\text { weight (mg) }\end{array}$} & \multicolumn{3}{|c|}{ Root:Shoot } & \multicolumn{3}{|c|}{$\begin{array}{l}\text { Amount of } \\
\text { respiration }\end{array}$} & \multicolumn{3}{|c|}{$\begin{array}{l}\text { Seed metabolic } \\
\text { efficiency }(\mathrm{g} / \mathrm{g})\end{array}$} \\
\hline & $\begin{array}{c}\text { Contro } \\
1\end{array}$ & $\begin{array}{l}\text { PEG } \\
15 \%\end{array}$ & $\begin{array}{l}\text { PEG } \\
25 \%\end{array}$ & $\begin{array}{c}\text { Contro } \\
1\end{array}$ & $\begin{array}{l}\text { PEG } \\
15 \%\end{array}$ & $\begin{array}{l}\text { PEG } \\
25 \%\end{array}$ & Control & $\begin{array}{l}\text { PEG } \\
15 \%\end{array}$ & $\begin{array}{l}\text { PEG } \\
25 \%\end{array}$ & Control & $\begin{array}{l}\text { PEG } \\
15 \%\end{array}$ & $\begin{array}{l}\text { PEG } \\
25 \%\end{array}$ \\
\hline BD-605 & 7.4 & 16.0 & 19.8 & 0.6 & 1.0 & 1.0 & 7.8 & 3.9 & 4.1 & 2.1 & 3.5 & 2.7 \\
\hline BD-607 & 5.4 & 13.3 & 17.3 & 0.6 & 1.1 & 0.9 & 9.0 & 3.7 & 3.3 & 1.7 & 3.8 & 3.1 \\
\hline BD-608 & 7.8 & 14.3 & 20.9 & 0.7 & 1.0 & 1.0 & 15.0 & 9.0 & 6.5 & 1.1 & 1.7 & 1.8 \\
\hline BD-610 & 10.6 & 23.7 & 25.2 & 0.5 & 0.7 & 0.7 & 11.9 & 3.9 & 2.5 & 1.1 & 2.9 & 3.5 \\
\hline BD-611 & 6.2 & 12.5 & 16.9 & 0.5 & 1.1 & 0.8 & 9.3 & 4.1 & 4.1 & 1.6 & 3.3 & 2.2 \\
\hline BD-612 & 5.0 & 12.7 & 17.9 & 0.6 & 1.0 & 1.4 & 16.0 & 10.2 & 4.6 & 1.0 & 1.3 & 3.0 \\
\hline BD-613 & 4.5 & 11.8 & 17.1 & 0.7 & 1.0 & 1.2 & 11.7 & 8.2 & 4.5 & 1.5 & 1.6 & 2.6 \\
\hline BD-614 & 6.4 & 9.1 & 22.3 & 0.7 & 0.9 & 0.6 & 13.5 & 10.0 & 3.7 & 1.1 & 1.5 & 3.0 \\
\hline BD-616 & 8.0 & 10.4 & 15.4 & 0.7 & 1.0 & 1.1 & 5.7 & 5.4 & 2.4 & 2.4 & 2.1 & 4.0 \\
\hline BD-617 & 5.1 & 9.9 & 18.2 & 0.6 & 1.0 & 1.2 & 16.4 & 11.5 & 7.7 & 0.9 & 1.3 & 1.4 \\
\hline BD-618 & 7.6 & 11.6 & 17.3 & 0.4 & 1.0 & 1.1 & 16.4 & 9.5 & 5.6 & 0.7 & 1.6 & 2.3 \\
\hline BD-622 & 5.4 & 12.5 & 24.0 & 0.5 & 0.8 & 0.4 & 15.2 & 8.3 & 2.0 & 0.9 & 1.6 & 3.9 \\
\hline BD-628 & 6.6 & 9.1 & 15.5 & 0.7 & 0.9 & 1.2 & 16.6 & 15.9 & 12.0 & 0.8 & 0.7 & 0.8 \\
\hline BD-629 & 4.4 & 11.6 & 17.0 & 0.4 & 1.2 & 1.4 & 12.6 & 5.4 & 3.1 & 1.0 & 2.4 & 3.4 \\
\hline BD-632 & 4.2 & 11.3 & 15.7 & 0.6 & 1.3 & 1.4 & 9.1 & 3.3 & 3.2 & 1.5 & 3.8 & 3.2 \\
\hline BD-633 & 6.5 & 14.9 & 20.2 & 0.6 & 0.8 & 1.0 & 18.6 & 7.6 & 6.3 & 0.7 & 2.1 & 2.0 \\
\hline BD-634 & 5.2 & 12.6 & 16.6 & 0.5 & 0.9 & 1.3 & 13.4 & 6.9 & 4.8 & 1.2 & 2.1 & 2.7 \\
\hline BD-635 & 5.2 & 14.6 & 18.1 & 0.5 & 0.8 & 1.0 & 17.0 & 10.5 & 9.8 & 0.9 & 1.2 & 1.0 \\
\hline BD-636 & 4.1 & 6.5 & 14.5 & 0.6 & 0.9 & 1.2 & 13.9 & 14.4 & 6.9 & 1.0 & 0.8 & 1.5 \\
\hline BD-638 & 5.3 & 18.5 & 24.8 & 0.5 & 0.5 & 1.1 & 15.3 & 6.7 & 3.9 & 1.1 & 1.7 & 2.6 \\
\hline BD-641 & 5.5 & 13.9 & 18.4 & 0.5 & 1.0 & 0.9 & 10.8 & 4.4 & 2.6 & 1.1 & 2.3 & 2.8 \\
\hline BD-642 & 13.0 & 18.4 & 23.5 & 0.5 & 1.1 & 0.6 & 16.2 & 11.9 & 9.1 & 0.8 & 0.9 & 1.0 \\
\hline BD-643 & 7.8 & 18.2 & 24.3 & 0.6 & 1.0 & 0.8 & 16.9 & 9.6 & 5.8 & 0.9 & 1.3 & 1.7 \\
\hline BD-644 & 8.2 & 16.7 & 16.9 & 0.5 & 0.8 & 1.0 & 13.9 & 7.5 & 6.8 & 1.0 & 1.5 & 1.3 \\
\hline BD-645 & 5.2 & 14.8 & 20.5 & 0.8 & 1.0 & 0.9 & 17.0 & 11.5 & 8.4 & 0.8 & 1.0 & 1.2 \\
\hline $\begin{array}{l}\text { LSD } \\
(0.05)\end{array}$ & & 2.59 & & & 0.34 & & & 0.45 & & & 0.53 & \\
\hline CV (\%) & & 10.14 & & & 19.45 & & & 2.58 & & & 15.66 & \\
\hline
\end{tabular}


Table 3. Effect of drought stress through polyethylene glycol (PEG-6000) solution on vigour index and relative vigour index of wheat genotypes.

\begin{tabular}{cccccccc}
\hline & \multicolumn{3}{|c|}{ Vigour index } & \multicolumn{2}{c}{ Relative vigour index } \\
\cline { 2 - 6 } Genotypes & Control & $15 \%$ PEG & $25 \%$ PEG & $15 \%$ PEG & $25 \%$ PEG \\
\hline BD-466 & 848 & 579 & 182 & -32 & -79 \\
BD-470 & 1265 & 686 & 350 & -46 & -72 \\
BD-473 & 1048 & 701 & 479 & -33 & -54 \\
BD-476 & 1163 & 662 & 300 & -43 & -74 \\
BD-479 & 1118 & 661 & 302 & -41 & -73 \\
BD-480 & 1195 & 1006 & 734 & -16 & -28 \\
BD-481 & 932 & 670 & 494 & -28 & -47 \\
BD-483 & 943 & 626 & 513 & -34 & -46 \\
BD-487 & 1083 & 770 & 261 & -29 & -76 \\
BD-488 & 1028 & 551 & 278 & -46 & -73 \\
BD-489 & 1188 & 805 & 485 & -32 & -59 \\
BD-491 & 1158 & 755 & 629 & -35 & -46 \\
BD-493 & 1302 & 916 & 554 & -30 & -57 \\
BD-496 & 1769 & 1191 & 926 & -33 & -48 \\
BD-497 & 1472 & 984 & 686 & -33 & -53 \\
BD-498 & 1169 & 944 & 838 & -19 & -28 \\
BD-499 & 1584 & 1340 & 1064 & -15 & -33 \\
BD-500 & 1415 & 808 & 840 & -43 & -41 \\
BD-501 & 1493 & 1352 & 1053 & -9 & -29 \\
BD-505 & 1434 & 1102 & 982 & -23 & -32 \\
BD-507 & 1399 & 802 & 945 & -43 & -32 \\
BD-508 & 1525 & 1139 & 966 & -25 & -37 \\
BD-509 & 1490 & 766 & 762 & -49 & -49 \\
BD-510 & 1737 & 1084 & 920 & -38 & -47 \\
BD-511 & 1406 & 824 & 889 & -41 & -37 \\
BD-512 & 1276 & 742 & 796 & -42 & -38 \\
BD-513 & 984 & 878 & 795 & -11 & -19 \\
BD-514 & 952 & 869 & 770 & -9 & -19 \\
BD-516 & 1485 & 859 & 897 & -42 & -40 \\
BD-517 & 1062 & 777 & 780 & -27 & -27 \\
BD-519 & 944 & 817 & 832 & -13 & -12 \\
BD-522 & 1217 & 895 & 843 & -26 & -31 \\
BD-524 & 1178 & 801 & 767 & -32 & -35 \\
BD-525 & 704 & 604 & 444 & -14 & -37 \\
BD-527 & 822 & 588 & 437 & -28 & -47 \\
\hline
\end{tabular}




\begin{tabular}{c|c|c|c|c|c}
\hline & \multicolumn{3}{|c|}{ Vigour index } & \multicolumn{2}{c}{ Relative vigour index } \\
\cline { 2 - 6 } Genotypes & Control & $15 \%$ PEG & $25 \%$ PEG & $15 \%$ PEG & $25 \%$ PEG \\
\hline BD-529 & 1306 & 605 & 792 & -54 & -39 \\
BD-531 & 1494 & 990 & 500 & -34 & -67 \\
BD-533 & 1231 & 906 & 611 & -26 & -50 \\
BD-534 & 1443 & 855 & 508 & -41 & -65 \\
BD-535 & 1355 & 646 & 397 & -52 & -71 \\
BD-536 & 1743 & 868 & 761 & -50 & -56 \\
BD-537 & 1500 & 686 & 563 & -54 & -62 \\
BD-539 & 1663 & 720 & 227 & -57 & -86 \\
BD-540 & 1411 & 733 & 323 & -48 & -77 \\
BD-541 & 1679 & 800 & 451 & -52 & -73 \\
BD-542 & 1062 & 746 & 334 & -30 & -69 \\
BD-545 & 1682 & 729 & 563 & -57 & -66 \\
BD-546 & 722 & 153 & 32 & -79 & -96 \\
BD-547 & 1556 & 792 & 660 & -49 & -58 \\
BD-551 & 937 & 249 & 20 & -73 & -98 \\
BD-552 & 1255 & 830 & 530 & -34 & -58 \\
BD-560 & 1337 & 899 & 452 & -33 & -66 \\
BD-562 & 1655 & 1041 & 703 & -37 & -58 \\
BD-563 & 1747 & 1128 & 672 & -35 & -62 \\
BD-567 & 1337 & 912 & 624 & -32 & -53 \\
BD-568 & 1538 & 716 & 587 & -53 & -62 \\
BD-569 & 1608 & 1029 & 543 & -36 & -66 \\
BD-570 & 1323 & 803 & 234 & -39 & -82 \\
BD-574 & 1278 & 949 & 269 & -26 & -79 \\
BD-576 & 1360 & 1041 & 512 & -23 & -62 \\
BD-579 & 1693 & 1118 & 388 & -34 & -77 \\
BD-581 & 1338 & 827 & 495 & -38 & -63 \\
BD-582 & 1632 & 1084 & 768 & -34 & -53 \\
BD-583 & 1627 & 1037 & 671 & -36 & -59 \\
BD-584 & 1462 & 1002 & 527 & -31 & -64 \\
BD-590 & 1295 & 618 & 496 & -52 & -62 \\
BD-592 & 1353 & 1232 & 970 & -9 & -28 \\
BD-593 & 1196 & 938 & 670 & -22 & -44 \\
BD-598 & 1554 & 1221 & 654 & -21 & -58 \\
BD-599 & 1527 & 1345 & 751 & -12 & -51 \\
BD-600 & 1725 & 1414 & 856 & -18 & -50 \\
BD-601 & 1593 & 1201 & 629 & -25 & -61 \\
\hline & & & & &
\end{tabular}




\begin{tabular}{c|c|c|c|c|c}
\hline & \multicolumn{3}{|c|}{ Vigour index } & \multicolumn{2}{c}{ Relative vigour index } \\
\cline { 2 - 6 } Genotypes & Control & $15 \%$ PEG & $25 \%$ PEG & $15 \%$ PEG & $25 \%$ PEG \\
\hline BD-602 & 1439 & 1196 & 843 & -17 & -41 \\
BD-603 & 1588 & 1318 & 889 & -17 & -44 \\
BD-604 & 1343 & 1116 & 760 & -17 & -43 \\
BD-605 & 1633 & 1208 & 811 & -26 & -50 \\
BD-607 & 1317 & 1037 & 610 & -21 & -54 \\
BD-608 & 1507 & 1190 & 719 & -21 & -52 \\
BD-610 & 1154 & 379 & 89 & -67 & -92 \\
BD-611 & 1332 & 1098 & 587 & -18 & -56 \\
BD-612 & 1433 & 1115 & 922 & -22 & -36 \\
BD-613 & 1562 & 1047 & 761 & -33 & -51 \\
BD-614 & 1313 & 1227 & 674 & -7 & -49 \\
BD-616 & 1283 & 963 & 621 & -25 & -52 \\
BD-617 & 1430 & 1137 & 624 & -20 & -56 \\
BD-618 & 1060 & 981 & 725 & -7 & -30 \\
BD-622 & 1302 & 1109 & 596 & -15 & -54 \\
BD-628 & 1190 & 825 & 461 & -31 & -61 \\
BD-629 & 1095 & 899 & 540 & -18 & -51 \\
BD-632 & 1308 & 1031 & 662 & -21 & -49 \\
BD-633 & 1230 & 1192 & 831 & -3 & -29 \\
BD-634 & 1433 & 1125 & 851 & -22 & -41 \\
BD-635 & 1593 & 998 & 701 & -37 & -56 \\
BD-636 & 1252 & 855 & 726 & -32 & -42 \\
BD-638 & 1532 & 965 & 491 & -37 & -68 \\
BD-641 & 972 & 719 & 435 & -26 & -55 \\
BD-642 & 940 & 788 & 521 & -16 & -45 \\
BD-643 & 1532 & 990 & 540 & -35 & -65 \\
BD-644 & 1118 & 781 & 437 & -30 & -61 \\
BD-645 & 1217 & 864 & 661 & -29 & -46 \\
LSD (0.05) & & 143.50 & & - & - \\
CV (\%) & & 7.65 & & - & - \\
\hline & & & & &
\end{tabular}

